\title{
DO DIFFERENCES IN EDUCATIONAL CULTURE AFFECT THE PROCESS AND OUTCOME OF UNDERGRADUATE DESIGN PRACTICE?
}

\author{
Ceri ALMROTT ${ }^{1}$, Dr Colm O’KANE ${ }^{1}$, Robert TULLY ${ }^{1}$ and Lyndon BUCK ${ }^{2}$ \\ ${ }^{1}$ Technological University Dublin, Republic of Ireland \\ ${ }^{2}$ Buckinghamshire New University, United Kingdom
}

\begin{abstract}
Designers undertake projects in different ways, and as might be expected, these have the potential to produce a wide variety of outputs. While the designed artefact is understandably often the focus when evaluating student designers, the importance of process is paramount in design education. This is underpinned by the fact that a novice designer may produce a single successful project without a full understanding of how that artefact came about. However, a designer who can reflect upon and understand the process, is better equipped to repeat or further evolve this process in future projects.

It is well understood that organisational structure and culture influences the ways designers choose to approach a project, from initial brief formation, through ideation and development, to the detail design and execution phases. An aspect not thoroughly understood is how different methods in design education specifically influence the methodologies designers employ in approaching a project, and therefore that projects outcome. Previous work has examined the influence of cultural background on the formation of a design brief, but not on the entire process.

This research attempts to understand that influence, by forming links between the educational background as an input and process and artefact as outputs. This paper proposes a research framework in which both artefact and process are examined. To this end, cultural probe type tools, which actively encourage design students to reflect upon and report upon their process during a design project, are used to allow student design practitioners to self-report their design process.
\end{abstract}

\section{Keywords: Design education, product design, design culture, design pedagogy}

\section{RESEARCH MOTIVATION AND GOAL}

Designers acknowledge that global organisations can have different ethos's and cultures which affect the way in which they undertake the process of design and the products that they develop [1]. It is also understood that different social cultures have and use value sets in different ways [2]. Branda, Silveira et al suggest that the way we educate students affects the way in which they interpret design briefs, and we can hypothesise that this also applies to other parts of design practice [3]. Together, these observations raise questions around the methods used to educate designers. Alternative approaches suggest themselves; students' tuition could be tailored to user preferences in their specific regions; or global practices could be incorporated into all pedagogies, regardless of location.

Clark [4] suggests that every institution, by virtue of its existence, has a particular culture or ethos, which is derived from the shared values, beliefs, knowledge and habits of the members of the institution. Ylijoki [5] argues that "disciplines have their own traditions and categories of thought which provide the members of the field with shared concepts of theories, methods, techniques and problems, as well as having their own social and cultural characteristics." These characteristics contribute to the formation of both personal and group identity and will influence the way students will potentially interpret the design brief.

This paper outlines a pilot study in which design practice across different institutions is analysed and assessed through ethnographic research processes and follow up interviews with students working on similar design briefs. By investigating the design processes deployed by individual students, the researchers aim to answer the following research questions in the context of a variation in cultural and educational experiences: 
Is there a commonality in the themes and influences during the research phase of a design project? How do students define their design problem? What tools and approaches do students use to conceptualise and develop their ideas? What approaches do students undertake to deliver their design concepts?

\section{METHODOLOGICAL APPROACH}

Several routes of investigation are possible including a historical look at past practice, as represented in student submissions of work for marking. By only referencing historical records (prior submissions) the researchers lose the ability to question the design students' intentions and allow clarity to be gained from reflective observation. Therefore, the approach must take in to account the ability to question a student's intended process and outcomes

Investigating practice and gaining insight into the reason why a route was taken in the design process requires a degree of self-reporting and reflection. Led questioning is also a key tool used to fully understand a designer's process. In turn, mapping findings from an institutional or cultural approach will likely require several layers of investigation to understand what (if any) core tools and processes are most implemented by a cohort of students and how much of an effect this might have on student work.

\subsection{Research approach}

In this research, we seek input from design students in the understanding of their practice. The iterative nature of the design process coupled with reflective questioning highlights the need for a participatory method [6] and makes an action research approach the most natural to take. By using the design student as a research subject, the voice of the student (the most important when looking at the influence teaching methods and syllabus subjects has on their practice) can be properly interrogated [7]. The findings of the research can lead to an improvement of the pedagogical methods implemented in the partner institutions [8]. This data-driven reflection on our influence as educators should allow for us to better craft student designers' educational experience.

By implementing a longitudinal study that looks at an individual designers' practice over a prolonged period, it is intended that maximum understanding of the way a designer uses tools and works through design problems can be captured. Coupling this with a study that runs through multiple phases of students over several years should also allow for a greater understanding of the nature of an educational institutions ability to influence and define a designer's process through the learning objectives and marking structures changing the emphasis of design education.

\subsection{Data collection}

Work at ISD Rubika [9] investigated how Cultural Probes could be used as a teaching tool to allow students to better empathise and understand self-reporting. It is proposed that a similar style of tool could be used by students to gather record and self-report on their design practice, as they are working. Subsequently further information is to be obtained from individual interviews with the research participant based on the data that they submitted during the probe process.

Cultural probes are a useful research tool for identifying and understanding the implications that a research participants cultural background may have on their actions [10]. Whilst the initial intention of a cultural probe is to provide design inspiration [11] by following the primary ethnographic stage with detailed follow up interviews enough data can be gathered to allow a good deal of insight into the research subjects thoughts and feelings [12]. This is particularly useful in the context of understanding design practice.

\subsection{Collaborative data}

Understanding the way in which colleagues in different schools as well as in different institutions practice a similar approach can be beneficial for both the teaching practitioner as well as for their students [13]. Educational programmes within the same institutions, taught across different schools can have similar outcomes but different emphasis in the formative stages of a design project will yield differing results. Both are likely to be considered correct but understanding the differing processes and their resulting effects upon the realisation of the artefact being produced can allow for a richer educational experience. Similarly, there is value in understanding how these differences affect outcomes across disparate institutions, both nationally and internationally. By partnering with different 
educational institutions and analysing the work of their students, a degree of understanding around their practice may be identified and shared.

\section{DESIGN AND IMPLEMENTATION}

There are a myriad number of factors that affect the outcome of a design project - from the formative influences and experiences of the designer to the resources and technical capabilities of the organisation. In many respects, there is little difference between an educational project scenario than an industrial one. The capabilities of the educational organisation will have as much of an effect on the outcome of the project as it would in a commercial setting. Through successive cycles of enquiry, patterns in practice in individual educational systems should become evident. These are likely to be shown in the outcomes of the design project and would result in similarities in project outcome being apparent in the work produced by students in different years. More conversely individual approaches and context should be shown across the work produced and there may be cross institutional themes perceptible in design outcomes for different cohorts of students. These would, in theory, reflect the zeitgeist of the industry at time of practice. By accurately and consistently measuring the tools used and the processes undertaken the impact of different programs on different cohorts will be able to be mapped and potentially, with a large enough study, wider cultural markers may be able to be identified.

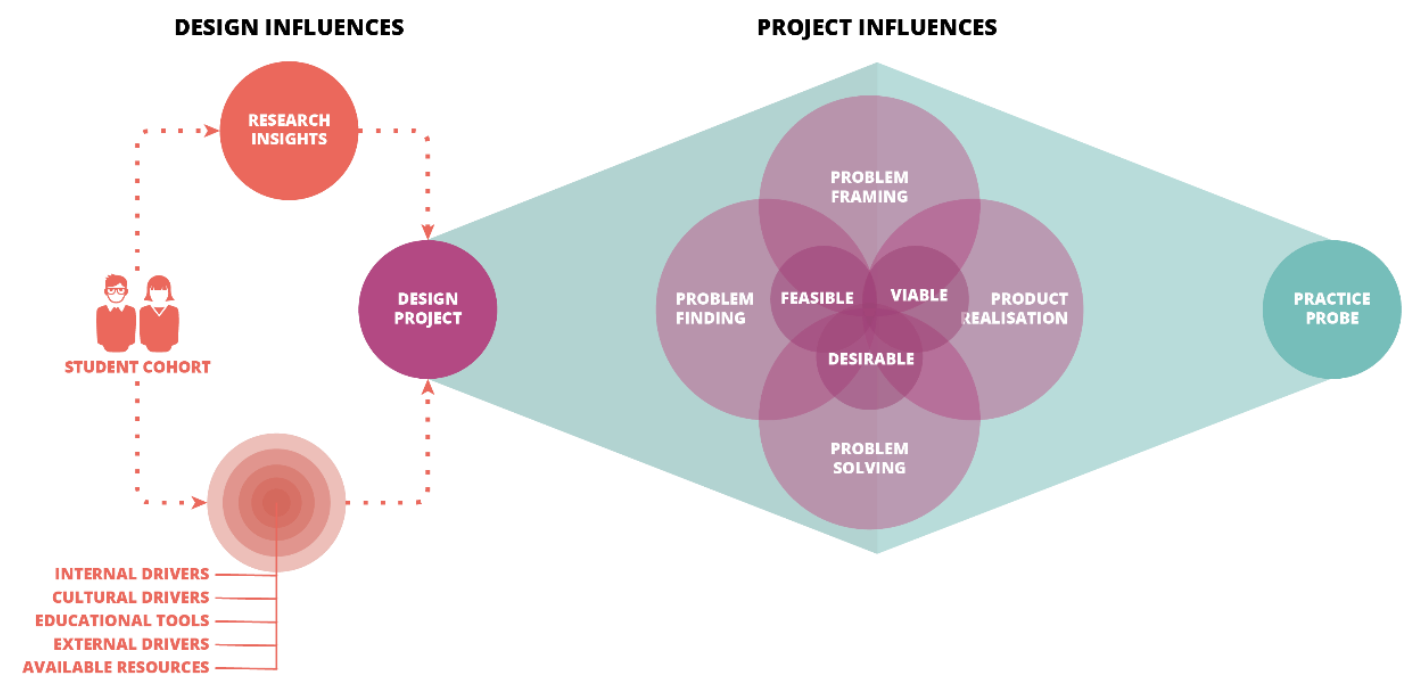

Figure 1. Aspects relating to the design process to be investigated using the Practice Probe

\subsection{Double diamond}

To frame the project and communicate it concisely with the research participants it was decided that the Design Council's double diamond model would act as a template for the development of the research tools. This description is a universally accessible aspect of design language which is used and referenced worldwide [14]. The questioning is mapped across the 4 regions of the double diamond to allow the research participant to understand how the lines of investigation relate to the different phases of Divergent and Convergent thinking described in the Design Council model. 


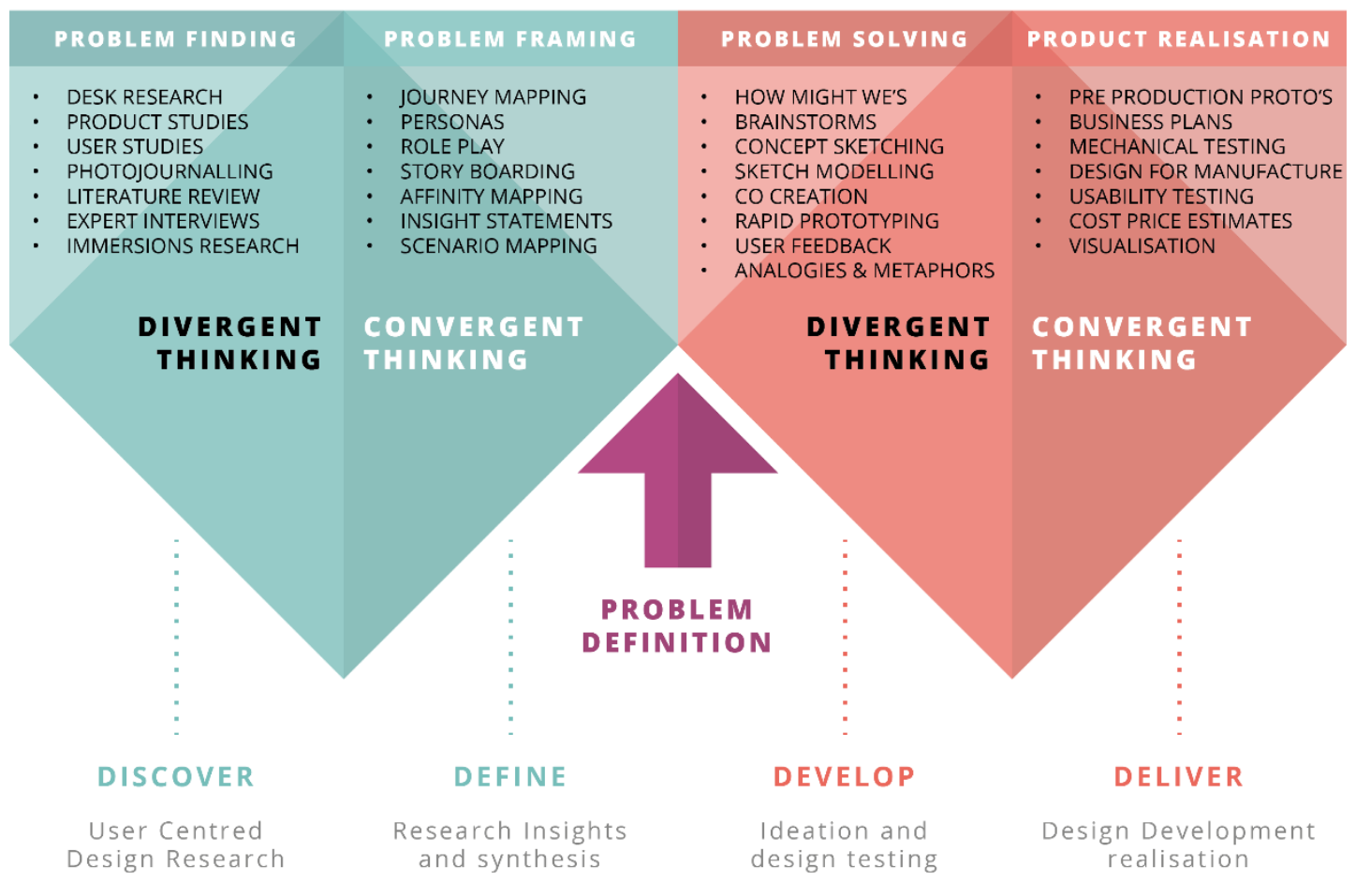

Figure 2. The Double Diamond mapped to design project tools

It is useful to understand how design practitioners from different contextual backgrounds structure their project around the user and the solution to the problem. The first diamond of the model is generally focused with understanding the context of the product use and creating enough empathy with the user to allow for a fully considered approach to the problem space. The second diamond is more solution focused and will consider the best approach to the problem definition. Understanding any significant difference in magnitude between these areas across different programs and different institutions will be worth understanding as this is likely to affect ongoing design practice.

\subsection{Research Tools}

The research is focussed on understanding what it is that influences and drives a designer to generate a solution to a given problem. The most basic of these questions can be broadly categorised in to "What has the designer done to understand the problem?" and "What has the designer done to create a solution to the problem?" We can simplify this down to User Focussed tasks and Solution focussed tasks. With a linear design process that moves from Problem to Solution the user focussed tasks should be loaded toward the front of the project and can be categorised in to the sub categories of Problem Finding and Problem Framing whilst the solution focussed tasks would ordinarily be found toward the end of the project and can be categorised as Problem Solving and Product Realisation.

Design activities that work within the four categories above can be both active (user interaction studies, prototype testing etc.) and passive (secondary research, material selection etc.). Depending on the type of student and the tools that are available for them to use it is likely that some students will be more likely to undertake passive tasks whereas others will be more comfortable undertaking active ones.

Research tools based on the Cultural Probe method rely on active participation of the participant in the generation of research assets that allow the researcher to gain deeper insight than traditional quantitative data would provide. A good probe tool, therefore, creates research activities that allow the research participant to want to engage in the study. Traditionally they have used tools such as sketching, photo journaling and voice notes to engage the participant and create a research asset which is wholly unique to them.

It was decided that a method of delivering the probe which had minimal impact on practice would be required as a traditional probe kit could alter process through duplication of work (sketchbook tasks 
etc). To do this a probe app was proposed and developed to allow students to report on their process. Although this approach has the unfortunate downside of removing the more designerly interaction that a physical probe provides it also provides the additional benefit of allowing a research activity to be answered in multiple ways. (Video, Voice Memo or written text for instance).

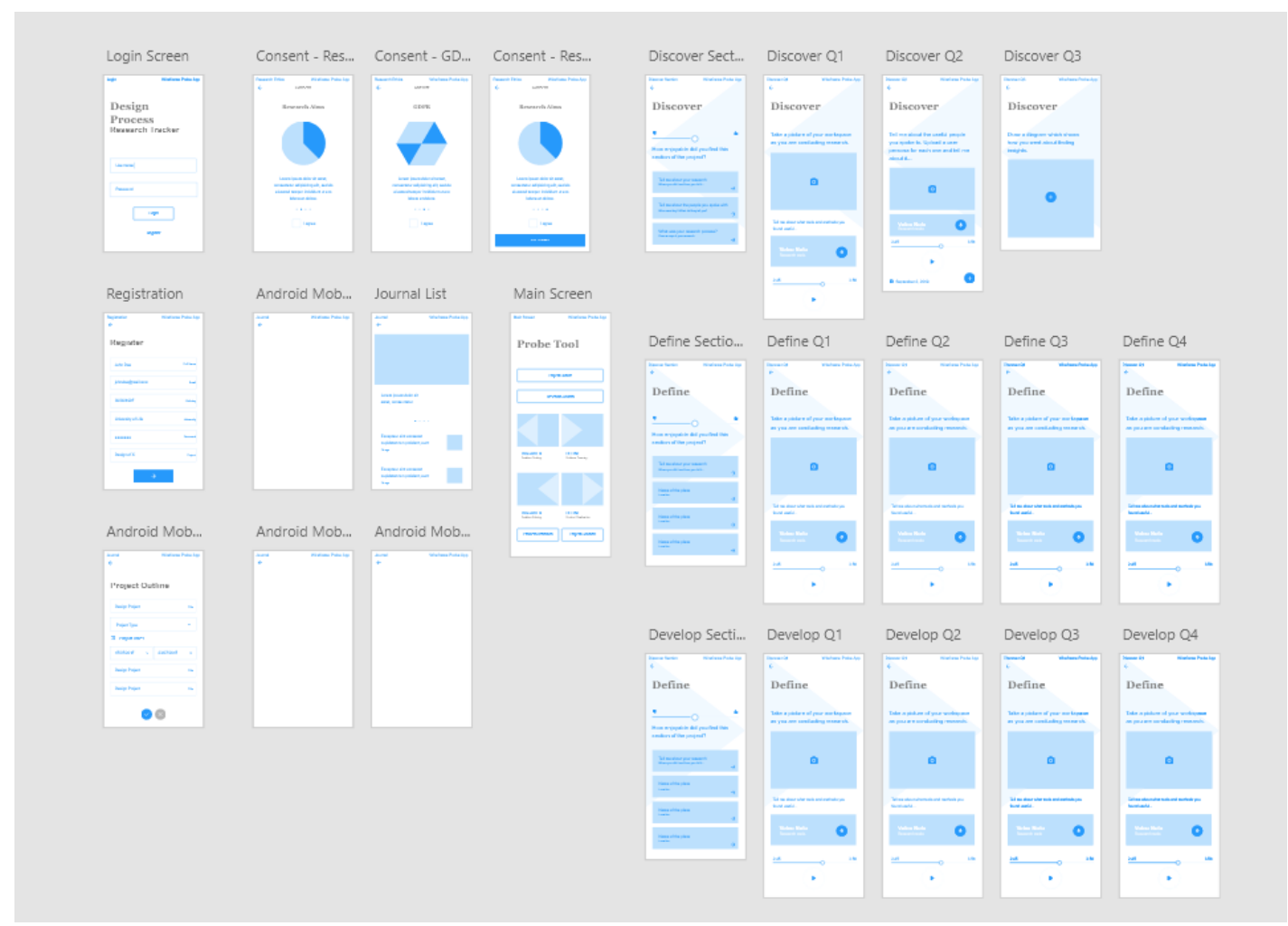

Figure 3. Wire frame design for the Practice Probe mobile application

On the completion of each cycle of study, researchers can view and thematically analyse the probe data to identify patterns of activity and influence across programmes and across disciplines. These themes will then provide the scaffolding for follow up interviews with each research participant to validate the findings and gain deeper specific insight. From this, insights can be drawn with respect to design practice. These insights can then be reported back to partner institutions and disseminated to the global design community.

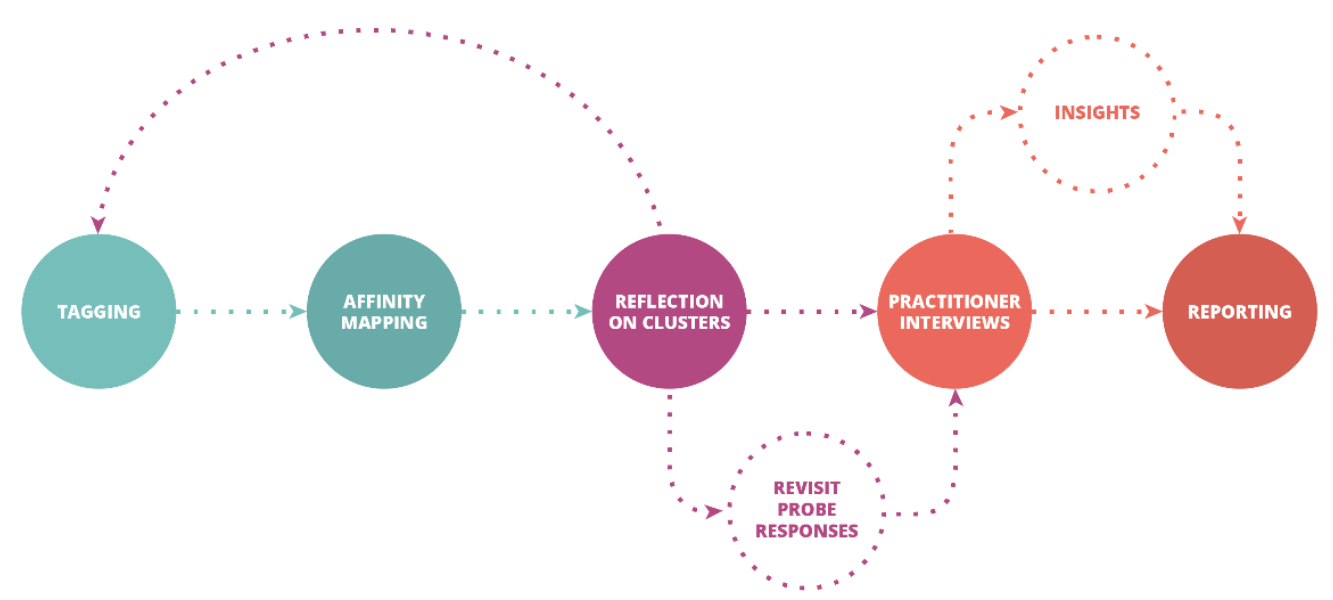

Figure 4. Process for interpreting the results from the Probe 


\section{CONCLUSIONS AND OPPORTUNITIES FOR FURTHER INVESTIGATION AND COLLABORATION}

Following from the initial pilot it is intended that the research will be continued and expanded across other institutions to allow a larger cross section of students, types of educational establishment and cultural context to be investigated. The study will be run cyclically for an ongoing period to allow those taking part to gain a greater understanding of how their pedagogical work is contextualised within the wider context of design education.

The intention is that this study can inform our understanding of the nature and influence of culture on professional identity in design practice. This may enable design pedagogy to construct appropriate design education interventions to navigate between local, regional and global contexts without eroding the richness of the individual designers' cultural identity

Furthermore, this student-led study will inform the design of a similar body of work undertaken as part of a doctoral programme. This second study will assess the cultural impact of design practitioners working on similar projects across disparate locations. The intention is to map and understand cultural markers within design practice with the intention of understanding global trends within our practice.

\section{REFERENCES}

[1] Karjalainen, T.M., Strategic design language - Transforming brand identity into Product Design elements, Proceedings of the 10th International Product Development Management Conference, 2003.

[2] Kotler, P. and Gertner, D. Country as brand, product, and beyond: A place marketing and brand management perspective, J. Brand Manag., vol. 9, no. 4, pp. 249-261, Apr. 2002.

[3] Branda, N., Silveira, L., van der Linden, J., Bohemia, E. and Kaygan, P. The design of the design brief: an analysis of Brazilian, Japanese and Turkish students, Proceedings of the 21st International Conference on Engineering and Product Design Education (E\&PDE 2019), University of Strathclyde, Glasgow. 12th -13th September 2019, 2019.

[4] Clark, B. R. The higher education system: academic organization in cross-national perspective. University of California Press, 1983.

[5] Ylijoki, O.H. Disciplinary cultures and the moral order of studying - A case-study of four Finnish university departments, High. Educ., vol. 39, no. 3, pp. 339-362, 2000.

[6] Price, R., Wrigley, C., Matthews, J. and Dreiling, A. Design research for the real world: A design-led innovation model for action research, Proceedings of NordDesign 2014 Conference, NordDesign 2014, 2014, pp. 154-163.

[7] Schulte, K. Ø., Hatling, M and Aschehoug, S.H. Improving innovation culture by demonstrator design, Proc. Nord. Nord. 2016, vol. 2, 2016.

[8] Liem, A., Ruecker, S. and Alfonso De La Rosa, J. Using studio teaching as an initiator and driver for research collaboration in design, Proceedings of the International Conference on Engineering Design, ICED, 2017, vol. 9, no. DS87-9, pp. 149-158.

[9] Green, C. Designing for self-reporting, Proceedings of the 21st International Conference on Engineering and Product Design Education (E\&PDE 2019), University of Strathclyde, Glasgow. 12th -13th September 2019, 2019, no. September.

[10] Gaver, B., Dunne, T. and Pacenti, E. Design: Cultural probes, interactions, vol. 6, no. 1, pp. 21-29, Jan. 1999.

[11] Gaver, B., Boucher, A., Pennington, S. and Walker, B. Cultural probes and the value of uncertainty, Interactions, 2004.

[12] van Leeuwen, J.P., Mayur Karnik, H. and Keane, K. Discovering Madeira: A Case Study of Cultural Probes, Proceedings of the DESIRE'11 Conference, Creativity and Innovation in Design: October 19-21, 2011, 2011.

[13] Bozalek, V. and Matthews, L. E-learning: A cross-institutional forum for sharing socio-cultural influences on personal and professional identity, Int. Soc. Work, vol. 52, no. 2, pp. 235-246, 2009.

[14] Ball, J. The Double Diamond: A universally accepted depiction of the design process, Design Council, 2019. [Online]. Available: https://www.designcouncil.org.uk/news-opinion /double-diamond-universally-accepted-depiction-design-process. [Accessed: 23-Feb-2020]. 